\title{
Análise da relação do produto interno bruto dos municípios do estado de Santa Catarina com as demonstrações do valor adicionado
}

\author{
Analysis of the relationship of gross domestic product of municipalities of Santa Catarina \\ state with added value statement
}

Análisis de la relación del producto interno bruto de municipios de Santa Catarina del estado con la declaración de valor añadido

\section{Larissa Degenhart}

Mestranda em Ciências Contábeis pela Universidade Regional de Blumenau

Endereço: Rua Antônio da Veiga, $n^{\circ}$ 140, Bairro Victor Konder

CEP: 89012-900 - Blumenau/SC - Brasil

E-mail: lari ipo@hotmail.com

Telefone: +55 (47) 3321-0565

\section{Mara Vogt}

Mestranda em Ciências Contábeis pela Universidade Regional de Blumenau

Endereço: Rua Antônio da Veiga, $n^{\circ}$ 140, Bairro Victor Konder

CEP: 89012-900 - Blumenau/SC - Brasil

E-mail: maravogtcco@gmail.com

Telefone: +55 (47) 3321-0565

\section{Nelson Hein}

Doutor em Engenharia de Produção pela Universidade Federal de Santa Catarina

Pós-Doutorado pelo IMPA - Associação Instituto Nacional de Matemática Pura e Aplicada e, pela Anderson School of Management da Universidade do Novo México

Professor do Departamento de Matemática da Universidade Regional de Blumenau e professor no Programa de Pós-Graduação em Ciências Contábeis (PPGCC) da Universidade Regional de Blumenau

Endereço: Rua Antônio da Veiga, $\mathrm{n}^{\circ}$ 140, Bairro Victor Konder

CEP: 89012-900 - Blumenau /SC - Brasil

E-mail: hein@furb.br

Telefone: +55 (47) 3321-0565

Artigo recebido em 22/07/2014. Revisado por pares em 12/11/2014. Recomendado para publicação em 01/12/2014 por Sandra Rolim Ensslin (Editora Científica). Publicado em $15 / 12 / 2014$. 


\title{
Resumo
}

Este estudo objetiva analisar a relação do Produto Interno Bruto (PIB) dos municípios do Estado de Santa Catarina com as Demonstrações do Valor Adicionado (DVA) do Agronegócio, Indústria, Serviços e Administração. Os resultados demonstram que houve uma forte relação do PIB com todas as DVAs analisadas, visto que todas se apresentaram significativas. Verificou-se ainda, que a DVA da Administração, quando analisada no conjunto foi a que menos apresentou correlação com o PIB e, na análise individual, a DVA do Agronegócio foi o que menos apresentou relação com o PIB. Além disso, estas duas variáveis são as que evidenciam menor valor, quando somados todos os valores de cada DVA, de todos os municípios e comparados às demais DVAs analisadas. Conclui-se que a DVA é uma forte aliada para a formação do PIB, diagnostica a situação dos municípios e evidencia o quanto estes estão colaborando para se chegar ao valor do PIB.

Palavras-chave: Produto Interno Bruto. Demonstração do Valor Adicionado. Santa Catarina.

\begin{abstract}
This study aims to analyze the relationship of Gross Domestic Product (GDP) of municipalities in the state of Santa Catarina with Added Value Statements (AVS) of Agribusiness Industry, Services and Administration. The results show that there was a strong relationship between GDP and all AVS analyzed since all were reported as significant. It was also found that the AVS of the Administration, when analyzed as a whole was the one which presented less correlation with GDP and in the individual analysis, the AVS Agribusiness was the one with less relation to GDP. Moreover, both variables are the ones showing lower value, when added all the values of each AVS, from all the municipalities and compared to other analyzed AVS. We conclude that the AVS is a strong ally for the formation of GDP, it diagnoses the situation of municipalities and shows how these are collaborating to reach the value of GDP.
\end{abstract}

Keywords: Gross Domestic Product. Added Value Statements. Santa Catarina.

\section{Resumen}

Este estudio tiene como objetivo analizar la relación del Producto Interno Bruto (PIB) de los municipios del Estado de Santa Catarina con las Demostraciones del Valor Añadido (DVA) del Agronegocio Industria, Servicios y Administración. Los resultados demuestran que hubo una fuerte relación del PIB con todas las DVAs analizadas, ya que todas se presentaron como significativas. También fue constatado, que la DVA de la Administración, al ser analizada en el conjunto fue la que menos presentó correlación con el PIB y, en el análisis individual, la DVA del Agronegocio fue la que presentó menos relación con el PIB. Además de eso, esta dos variables son las que muestran menor valor, al ser sumados todos los valores de cada DVA, de todos los municipios y comparados a las demás DVAs analizadas. Concluimos que la DVA es una fuerte aliada para la formación del PIB, diagnostica la situación de los municipios y muestra cuanto estos están colaborando para llegar al valor del PIB.

Palabras Clave: Producto Interno Bruto. Demonstración del Valor Añadido. Santa Catarina. 


\section{Introdução}

O cenário mundial vem sendo caracterizado por significativas mudanças na área social, política e econômica, refletindo no ambiente empresarial, por meio da imposição de novos padrões de competitividade, perante a necessidade de alterar as práticas relacionadas ao trabalho e a gestão da empresa. Deste modo, a informação contábil tradicional contemplada nos relatórios contábeis clássicos, não está abrangendo totalmente as novas demandas dos usuários dessa informação (COSENZA, 2003).

Neste contexto, Burchell, Clubb e Hopwood (1985) salientam que a contabilidade esta começando a ser vista como um bem social e não apenas como um fenômeno voltado a técnicas, pois a contabilidade passou a ser mais ativa e reconhecida como um instrumento que auxilia na gestão social voltada para a mudança.

Neste sentido, a contabilidade como fornecedora de informações, segundo Cunha, Ribeiro e Santos (2005), possui um grande desafio de colocar a disposição dos usuários da informação contábil, informações que demonstram as relações da empresa para com a sociedade. Assim, a Demonstração do Valor Adicionado (DVA), apresenta-se como um dos instrumentos capazes de evidenciar os aspectos econômicos e sociais, inovando o enfoque que foi utilizado até então pela contabilidade, constituindo uma das mais ricas demonstrações contábeis. Para tanto, de acordo com Bao e Bao (1998), o valor econômico adicionado, passa a ser um substituto para o lucro econômico, recebendo muita atenção dentre as demonstrações contábeis, por ser uma medida voltada para o desempenho da empresa.

Contudo, é inegável que maior comprometimento da empresa para com os aspectos sociais, tende a agregar valor, sendo de grande importância para a sociedade, a existência de uma ferramenta que possibilite avaliar o grau de comprometimento da empresa com os problemas sociais. Deste modo, a DVA tem sido apontada como um dos instrumentos mais significativos que foi incorporada no leque das demonstrações contábeis obrigatórias, por meio da Lei $\mathrm{n}^{\mathrm{o}} 11.638 / 07$, em grande parte, devido a seu viés voltado para a parte social (SIQUEIRA, 2007).

Alexander e Buchholz (1978) afirmam que as empresas que estão preocupadas com os aspectos sociais, apresentam reflexos positivos nos tradicionais indicadores de desempenho, fazendo com que os investimentos sejam mais atrativos. Segundo Ullmann (1985) esse reflexo pode ocorrer também no preço das ações e ainda, passar a diminuir os índices de risco para efetuar os investimentos.

Luca (1998) destaca que a DVA, é um relatório contábil, que apresenta um conjunto de informações de natureza econômica, com o objetivo de evidenciar o valor da riqueza gerada pela empresa e a sua distribuição para as partes que contribuíram para a geração desse valor. Aswegen, Steyn e Hamman (2005), frisam que o valor adicionado é uma medida da riqueza que foi criada por meio das atividades da empresa, sendo possível avaliar o desempenho destas.

Já para Morley (1979), a utilização dos índices baseados no valor adicionado, proporciona diagnosticar a situação da empresa. Para tanto, a DVA é reconhecida na contabilidade, por ser de fácil compreensão entre os diversos usuários da informação contábil (MEEK; GRAY, 1998; STAINBANK, 2009).

De acordo com Marion (1999), a DVA evidencia os componentes que geram o valor adicionado, ou seja, quanto de riqueza uma empresa produziu e de que forma foi distribuída 
aos empregados, acionistas, financiadores, governo e ainda, quanto ficou retido na própria empresa. É uma demonstração útil, pois o somatório de valores adicionados do país representa o seu PIB. Boullosa e Tavares (2009) destacam que o Produto Interno Bruto (PIB) é um índice macroeconômico de mensuração das atividades econômicas de certa região, município, Estado, país durante um período que pode ser mensal, trimestral e anual.

Diante disso, a Demonstração do Valor Adicionado vem ganhando importância de uma forma global, sendo um tema de constante debate na literatura contábil, pois diversos estudos como o de Bentley (1981), Burchell, Clubb e Hopwood (1985), Chua (1977), Pendrill (1977), Van Staden (1998) Evaert e Riahi-Belkaoui (1998) e Pong e Mitchell (2005) analisaram a utilidade da DVA, como uma ferramenta de análise. Contudo, não foram encontrados estudos que buscaram analisar a relação do PIB com a DVA, identificando uma lacuna de pesquisa.

Frente ao exposto, destaca-se a seguinte questão que norteia o desenvolvimento desta pesquisa: qual a relação do Produto Interno Bruto dos municípios do Estado de Santa Catarina com a Demonstração do Valor Adicionado? Com o intuito de responder a questão apresentada, o objetivo deste estudo é analisar a relação do Produto Interno Bruto dos municípios do Estado de Santa Catarina com as Demonstrações do Valor Adicionado.

Esta pesquisa justifica-se conforme Pinto e Ribeiro (2004), pois, acredita-se que a divulgação das ações sociais das empresas, dos planos voltados ao seu ativo humano, as políticas de investimento ao meio ambiente, bem como, a distribuição da riqueza e contribuição da empresa para com entidades assistenciais apresentam-se como de grande utilidade tanto para os usuários da informação contábil, quanto para a empresa que publica essas informações, pois a DVA é um instrumento que possibilita melhorar a imagem da empresa, além de servir como uma ferramenta de controle e avaliação gerencial.

Portanto, a relevância do estudo ampara-se em Martins et al. (2013), pois dentre os principais indicadores socioeconômicos que relaciona-se diretamente com a Demonstração do Valor Adicionado têm-se o Produto Interno Bruto, sendo que está relação se dá em função do cálculo do PIB ser semelhante ao cálculo do valor adicionado. Nesse sentido, o PIB é a totalidade de renda obtida internamente em relação a um país, Estado. Já o valor adicionado refere-se à totalidade de renda que foi obtida de forma interna na empresa.

\section{Referencial Teórico}

$\mathrm{Na}$ revisão de literatura são abordados aspectos que visam explorar a relação do Produto Interno Bruto com a Demonstração do Valor Adicionado, estes que norteiam o desenvolvimento e as premissas para consecução do estudo. Inicialmente destaca-se a importância do Produto Interno Bruto. Na sequência aborda-se a relevância da Demonstração do Valor Adicionado.

\subsection{Produto Interno Bruto - PIB}

A mensuração do Produto Interno Bruto, o denominado PIB, para qualquer análise econômica é considerado de grande valia, sendo que as diferenças regionais que existem, principalmente em países como o Brasil, devem-se em função do descaso do governo, por não 
considerarem importante o dimensionamento do PIB, para a formulação das políticas públicas (ARAÚJO NETO; COSTA, 2005).

Conforme Furtuoso e Guilhoto (2003) o PIB é considerado um indicador econômico fundamental, pois atua na forma de subsídio, base, para os formadores de políticas econômicas, bem como, para os tomadores de decisão tanto no âmbito governamental, quanto privado. Araujo, Fajardo e Tavani (2006) salientam que o PIB é o resultado de todos os fatores e atividades da economia, ou seja, inclui-se o capital humano, o mercado financeiro, os imóveis e ainda, qualquer mercado significativo.

Sachs e Larrain (2000) frisam que o PIB é o valor total da produção atual de produtos e serviços finalizados que são obtidos no território nacional, em determinado período, geralmente de um ano e até mesmo trimestre. Já para Gastaldi (2005), é o valor de todos os bens e serviços que são produzidos nos países, em um período de um ano e considerados a preços correntes de mercado.

Diante disso, Rossetti (2003) destaca que o PIB considera a produção que ocorre nas fronteiras econômicas do território nacional, seja gerada por recursos internos do país ou do exterior, ou seja, é o total do valor adicionado bruto pelas empresas, com a inclusão dos impostos indiretos líquidos. O autor salienta ainda, que o PIB é um dos indicadores mais utilizados na macroeconomia, pois objetiva mensurar a atividade econômica de uma determinada região, sendo o principal indicador utilizado para evidenciar a geração da riqueza e o crescimento econômico.

Para Heilbroner e Thurow (2001), a produção medida pelo PIB, apresenta relação com o nível de emprego e com a renda a ser recebida da população, sendo um dos índices utilizados para medir o desenvolvimento humano. Deste modo, Simonsen (1979) conceitua o PIB como sendo o valor bruto de toda a produção, menos os consumos intermediários. Assim, é concebido como a soma dos valores adicionados, em todas as etapas do processo de produção de determinado país. Dessa forma, para se apurar o produto nacional de uma economia, é necessário somar todos os valores adicionais relativos a cada etapa de produção.

Nesse contexto, Santos e Hashimoto (2003), destacam que a elaboração da Demonstração do Valor Adicionado pelas empresas possibilita gerar informações para subsidiar a tarefa de se estimar o PIB de determinado país, pois essa demonstração é utilizada na economia, macroeconomia, para definir e calcular o denominado produto nacional, que está ligado a possível riqueza gerada pelo sistema econômico.

Segundo o CPC 09 (2008), a DVA busca apresentar a parcela que a entidade contribuiu para a formação do Produto Interno Bruto. Entretanto, existem diferenças entre os modelos contábil e econômico, para a realização do cálculo do valor adicionado, pois o modelo econômico toma por base a produção para o cálculo do PIB, já a contabilidade emprega o conceito contábil da realização da receita, ou seja, baseia-se no regime contábil de competência. Nesse sentido, como a produção e a venda acontecem normalmente em períodos diferentes, consequentemente os valores utilizados para o cálculo do PIB tanto para o modelo contábil quanto para o modelo econômico serão diferentes em cada período.

Vale ressaltar que a metodologia de cálculo para o Produto Interno Bruto, requer cuidados, pois junta diferentes indicadores. De acordo com Marion (2001, p. 67),

o somatório dos valores adicionados (ou valores agregados) de um país representa o seu Produto Interno Bruto (PIB), sendo que essa informação é tão importante que, além de sua utilização pelos países europeus, alguns países emergentes só aceitam a 
instalação e a manutenção de uma empresa transnacional se ela demonstrar qual será o valor adicionado que irá produzir.

Conforme Ioan (2014), a harmonização dos empregados, empregadores e o Estado, envolve a construção dos sistemas de distribuição de valor agregado, bruto, bem como, líquido, principalmente o PIB, pois o sucesso da distribuição deste é um fator determinante para o real papel dos impostos na política econômica. Diante disso, Pinto e Ribeiro (2004), enfatizam que está sendo exigido das empresas, contribuir para com o desenvolvimento econômico e sustentável da nação, condicionando uma demonstração que realiza a devida contribuição, ou seja, a elaboração da DVA.

Contudo, diversos foram os estudos realizados com o Produto Interno Bruto, no intuito de entender como os gastos públicos podem elevar o crescimento econômico, devido o aumento da produtividade do setor privado (RAM, 1989; BARRO, 1990; CASHIN, 1995; ASCAHUER, 1989). No entanto, o valor adicionado também é objeto de estudo da macroeconomia, principalmente no que tange a avaliação e entendimento do Produto Interno Bruto.

\subsection{Demonstração do Valor Adicionado - DVA}

A Demonstração do Valor Adicionado, conforme Knell (1986) surgiu da necessidade de algumas companhias europeias constituírem melhor a relação existente com seus acionistas. Em relação aos empregados, o objetivo dessa demonstração era conseguir maior motivação e consequentemente produtividade.

Para Luca (1998) esta demonstração relaciona-se com o conceito de responsabilidade social e surgiu na área econômica com o propósito de atender às necessidades relacionadas às informações dos usuários sobre o valor da riqueza gerada pela empresa e sua devida utilização. Conforme Haller e Stolowy (1998), o valor adicionado é uma medida do desempenho econômico das empresas e possui uma vasta história de aplicação na área da economia.

Deste modo, Evraert e Riahi-Belkaoui (1998) destacam que baseado na Demonstração do Valor Adicionado, qualquer grupo de interesse poderá conhecer de fato, qual a riqueza gerada pelas empresas e sua distribuição entre os agentes econômicos, diferenciando-se da Demonstração do Resultado do Exercício, esta que objetiva informar a riqueza apenas criada sob a ótica do proprietário.

Neste contexto, manifesta-se a relevância das empresas apresentarem uma demonstração econômico-financeira que propicie informações compreensíveis e transparentes a todos os usuários da informação contábil. Desse modo, a DVA assume o papel de demonstração contábil complementar, com o intuito de informar sobre a participação dos fatores econômicos que interferiram na criação de valor para a entidade e ainda, evidencia a distribuição desse valor entre os agentes econômicos da organização (COSENZA, 2003).

Luca (1998) salienta que a Demonstração do Valor Adicionado é um conjunto de informações de natureza econômica, disponibilizadas em um relatório contábil, visando demonstrar a riqueza gerada pela empresa e a alocação desse valor, para os componentes que contribuíram no processo. Essa demonstração possibilita a avaliação das condições do Estado em proporcionar os benefícios à sociedade com base nos impostos arrecadados, avaliar a contribuição da empresa para a região, se a empresa está conseguindo comprar e vender mercadorias, bens, gerando assim, riquezas para a comunidade em que está inserida. 
O valor adicionado, ou valor agregado como também pode ser denominado, é utilizado para a avaliação do Produto Nacional, ou seja, a medida em unidades monetárias, do fluxo total dos bens e serviços finalizados que foram produzidos pelo sistema econômico em determinados períodos (LUCA, 1998). Segundo o Accounting Standards Steering Committee (ASSC) (1975) o valor adicionado é a riqueza criada por conta própria pelas empresas juntamente com os seus funcionários, evidenciando o valor adicionado que foi utilizado para pagar as partes que contribuíram para a sua devida criação.

De acordo com Haller e Stolowy (1998), o valor agregado é considerado uma medida do desempenho econômico, sendo reconhecido como um incremento na riqueza das entidades econômicas. As informações que constam no valor adicionado não se referem apenas aos aspectos econômicos, mas ao âmbito social também, identificando a parcela de participação de cada uma das partes no processo, revelando o resultado da empresa. Conforme Tinoco (1984) o valor adicionado contábil pela empresa para a sociedade é a diferença entre o valor das vendas totais e o valor das compras de bens, mercadorias e serviços.

Riahi-Belkaoui (1998) ressaltam que o valor adicionado se refere ao incremento na riqueza gerada, por meio da utilização dos recursos da empresa, antes de ocorrer a sua alocação aos acionistas, credores, funcionários, bem como, o governo. Deste modo, enquanto que o lucro é o retorno final desejado pelos acionistas, o valor adicionado refere-se ao retorno que os trabalhadores, provedores de capital e o governo receberão. Nesse sentido, a contabilidade realiza o cálculo do valor adicionado, por meio da diferença entre o valor oriundo da receita bruta e o valor que foi utilizado para adquirir os bens, mercadorias.

Segundo Kroetz (2000) por meio da Demonstração do Valor Adicionado é possível identificar a contribuição econômica da entidade em relação a cada segmento com o qual a empresa esta relacionada, constituindo-se no PIB produzido pela entidade.

Suojanen (1954) frisa que a DVA é considerada um relatório complementar de análise do valor agregado na produção, pois a metodologia para se encontrar o valor adicionado parte de um conceito econômico de renda, enfatizando a produção como sendo a medida do desempenho social das entidades. Bao e Bao (1998) salientam que a DVA busca medir a contribuição que uma empresa consegue possibilitar para com a sociedade, pois evidencia como os benefícios das empresas foram distribuídos entre as partes interessadas.

A evidenciação da geração e distribuição do valor adicionado ocorreu a partir da publicação da DVA, esta que foi regulamentada na legislação Brasileira, com a instituição da Lei $\mathrm{n}^{\circ} 11.638 / 07$, por meio do artigo 176 inciso $\mathrm{V}$, que torna obrigatória essa demonstração para as companhias abertas, como parte das demonstrações contábeis que são divulgadas no final de cada exercício (MARTINS et al., 2013).

Conforme o CPC 09 (2008), a DVA fundamenta-se em conceitos macroeconômicos e visa apresentar a parcela de contribuição que a entidade apresenta, perante a formação do PIB. Essa demonstração evidencia o quanto a entidade agrega valor aos insumos que são adquiridos de terceiros e que posteriormente são vendidos e até mesmo consumidos durante certo período, porém, existem diferenças em relação ao modelo contábil e econômico, para com o cálculo do valor adicionado. Deste modo, a ciência econômica em relação ao cálculo do PIB, baseia-se na produção, enquanto que a contabilidade emprega o conceito contábil da realização da receita, ou seja, toma por base o regime contábil de competência.

Segundo Burchell, Clubb e Hopwood (1985), a ambiguidade do valor adicionado, de certo modo, implicou seu funcionamento, sendo visto como um fator determinante para o processo da mudança social. Para tanto, a contabilidade é vista como um bem social e não 
apenas designada como uma técnica, passando dessa forma a ser mais ativa, bem como, reconhecida como um instrumento de gestão social que promove mudanças na sociedade. Contudo, Picur (2007) destaca que as omissões das divulgações relacionadas ao valor adicionado, podem passar a afetar o processo de tomada de decisão, sendo assim, muito importante para as empresas.

\section{Procedimentos Metodológicos}

Diante do objetivo de analisar a relação do Produto Interno Bruto dos municípios catarinenses com as Demonstrações do Valor Adicionado, realizou-se uma pesquisa descritiva, de corte seccional e quantitativa.

A pesquisa é caracterizada como descritiva, pois expõe as características de determinada população ou fenômeno. Além disso, pode estabelecer correlações entre as variáveis e definir sua natureza, não tendo o compromisso de explicar os fenômenos na qual descreve, mesmo que estes sirvam de base (VERGARA, 1998).

No que se refere ao tempo em que os dados foram coletados, este estudo apresenta um corte transversal (cross sectional) que para Sampieri et al. (1991, p. 2), esse corte ocorre quando "a coleta de dados ocorre em um só momento, pretendendo descrever e analisar o estado de uma ou mais variáveis em um dado momento".

O estudo caracteriza-se ainda como quantitativo, pois "envolve coleta, classificação, sumarização, organização, análise e interpretação de dados" para que na sequência, o pesquisador possa tratar os dados por meio da aplicação de métodos e técnicas estatísticas, procedendo assim, a análise e interpretação destes dados (MARTINS; THEÓPHILO, 2009, p. 107).

O período do estudo compreende o ano de 2010, ano do último Censo Demográfico divulgado pelo Instituto Brasileiro de Geografia e Estatística (IBGE). A população e amostra do estudo compreendem todos os municípios de Santa Catarina, ou seja, 293 municípios.

Os dados foram coletados no mês de abril de 2014 direto no sítio do IBGE, por meio de uma planilha eletrônica de excel e referem-se ao Produto Interno Bruto a preços correntes e ao Valor Adicionado Bruto da Agropecuária, Indústria, Serviços e da Administração, os quais foram relacionados com o PIB por meio da regressão linear múltipla a partir do software estatístico LHStat ${ }^{\circledR}$.

Optou-se por esse método estatístico, pois conforme Hair Jr. et al. (p. 32, 2005) "regressão múltipla é o método de análise apropriado quando o problema de pesquisa envolve uma única variável dependente métrica considerada relacionada a duas ou mais variáveis independentes métricas". Neste estudo, considera-se a variável independente o Produto Interno Bruto (PIB) e as variáveis dependentes são as quatro Demonstrações do Valor Adicionado (DVA) analisadas. Assim, este método objetiva prever as mudanças na variável dependente, sendo as respostas aa mudanças nas variáveis independentes.

\section{Descrição e Análise dos Dados}

Nesta seção, apresenta-se a descrição e análise dos dados, em que analisou-se a relação do Produto Interno Bruto com as Demonstrações do Valor Adicionado da Agropecuária, 
Indústria, Serviços e Administração. Inicialmente realizou-se uma estatística descritiva das variáveis utilizadas no estudo, estas que apresentam-se em valores monetários e, na sequência foram feitos os testes de aleatoriedade, normalidade e homocedasticidade, para então executar a regressão linear múltipla. Por meio da Tabela 1 descreve-se a estatística descritiva.

Tabela 1 - Estatística Descritiva (em R\$)

\begin{tabular}{l|c|c|c|c|c}
\hline \multicolumn{1}{c|}{ Variáveis } & Mínimo & Máximo & Média & Mediana & Desvio Padrão \\
\hline PIB & $18.006,51$ & $18.473 .989,58$ & $520.417,54$ & $123.937,10$ & $1.694 .572,71$ \\
\hline DVA AGRO & $2.291,13$ & $188.583,00$ & $29.876,57$ & $20.854,13$ & $28.264,40$ \\
\hline DVA IND & $1.565,33$ & $8.281 .401,44$ & $151.972,36$ & $23.643,91$ & $565.303,75$ \\
\hline DVA SERV & $8.732,43$ & $8.057 .681,57$ & $263.945,94$ & $54.119,65$ & $888.122,71$ \\
\hline DVA ADM & $5.001,32$ & $1.253 .950,06$ & $51.154,15$ & $17.853,20$ & $123.871,53$ \\
\hline
\end{tabular}

Fonte: Dados da pesquisa.

Conforme os dados da Tabela 1 observa-se que o valor mínimo do Produto Interno Bruto (PIB) foi de $\mathrm{R} \$ 18.006,51$ e seu valor máximo foi de $\mathrm{R} \$ 18.473 .989,58$. A média encontrada nessa variável foi de $\mathrm{R} \$ 520.417,54$ e a mediana correspondeu a $\mathrm{R} \$ 123.937,10$. Além disso, nota-se que o desvio padrão apresentou valor elevado em relação à média, sendo este de R\$1.694.572,71.

Quanto a Demonstração do Valor Adicionado da Agropecuária (DVA AGRO), verifica-se que o menor e maior valor apresentado é de $\mathrm{R} \$ 2.291,13$ e $\mathrm{R} \$ 188.583,00$ reais respectivamente. A média apresentou um valor de $\mathrm{R} \$ 29.876,57$ e a mediana de $\mathrm{R} \$$ 20.854,13. O desvio padrão para essa variável evidenciou uma diferença de $\mathrm{R} \$ 1.612,17$, ficando abaixo do valor da média.

Na sequência, analisando-se a Demonstração do Valor Adicionado da Indústria (DVA IND) percebe-se que o mínimo compreendeu um valor de $\mathrm{R} \$ 1.565,33$ e o máximo foi de $\mathrm{R} \$$ 8.281.401,44 e, percebe-se que a diferença foi a maior entre ambos os valores, quando comparado às diferenças entre o mínimo e máximo das demais DVAs apresentadas. A média desta variável foi de $\mathrm{R} \$ 151.972,33$ e a mediana de $\mathrm{R} \$ 23.643,91$. Além disso, verifica-se que o desvio padrão foi de R $\$ 565.303,75$.

Analisando-se a Demonstração do Valor Adicionado dos Serviços (DVA SERV) verifica-se que o valor mínimo encontrado foi de $\mathrm{R} \$ 8.732,43$ e o máximo $\mathrm{R} \$ 8.057 .681,57$. Além disso, a média apresentou-se bem abaixo do desvio padrão com uma diferença de R\$ $624.176,77$, sendo a maior diferença encontrada se comparado às demais DVAs analisadas no estudo.

A variável Demonstração do Valor Adicionado da Administração (DVA ADM) apresentou um valor mínimo e máximo de $\mathrm{R} \$ 5.001,32$ e $\mathrm{R} \$ 1.253 .950,06$ respectivamente. A média foi de $\mathrm{R} \$ 51.154,15$ e a mediana de $\mathrm{R} \$ 17.853,20$ no período analisado. Por fim, o desvio padrão evidenciou um valor de $\mathrm{R} \$ 123.871,53$.

Antes de realizar a regressão linear múltipla, destaca-se o atendimento aos pressupostos básicos para a utilização da técnica de regressão previstos de acordo o que Kennedy (1998) estabelece, sendo os testes de aleatoriedade, normalidade e de homocedasticidade apresentados conforme a Tabela 2.

De acordo com a Tabela 2 verificou-se que o Durbin-Watson do modelo ficou próximo a 2, ou seja, a independência dos erros é satisfeita e demonstra que inexiste autocorrelação entre os resíduos (MAROCO, 2011). Conforme Fávero et al. (2009, p. 357), "quando existe um problema de autocorrelação entre os resíduos deve-se ao fato de o resíduo 
incorporar os efeitos de uma variável que foi excluída do modelo, sua existência pode ser verificada por meio de Durbin-Watson".

Tabela 2 - Testes de aleatoriedade, normalidade e homocedasticidade

\begin{tabular}{l|l|l}
\hline \multicolumn{1}{c|}{ Pressupostos } & \multicolumn{1}{|c}{ Testes } & \multicolumn{1}{c}{ Hipótese } \\
\hline \multirow{2}{*}{ Aleatoriedade } & $\begin{array}{l}\text { Durbin-Watson: } \\
\text { Estatística DW }=1,9749 \\
\text { Valor-p }=0,5975 \\
\text { Autocorrelação dos resíduos }=0,0126\end{array}$ & $\begin{array}{l}\text { Inexiste autocorrelação de primeira ordem } \\
\text { entre os resíduos. }\end{array}$ \\
\hline \multirow{2}{*}{ Normalidade } & $\begin{array}{l}\text { Shapiro-Wilk: } \\
\text { Estatística } \mathrm{SW}=0,4650 \\
\text { Valor-p }=0,0000\end{array}$ & A distribuição dos resíduos é normal. \\
\hline \multirow{2}{*}{ Homocedasticidade } & $\begin{array}{l}\text { Levene: } \\
\text { Estatística } \mathrm{F}=0,9694 \\
\text { Valor-p }=0,3257\end{array}$ & A variância dos erros é uniforme. \\
\hline
\end{tabular}

Fonte: Dados da pesquisa.

Além disso, efetuou-se o teste Shapiro-Wilk para verificar a normalidade dos dados, que segundo Hair Jr. et al. (2009) representa o grau de distribuição dos dados. Dessa forma, constatou-se que a distribuição dos resíduos é normal, devido à sua significância ao nível de $1 \%$. Realizou-se ainda o teste de Levene para verificar a homocedasticidade que, segundo Hair Jr. et al. (2009) representa a igualdade de variância e, nota-se que a variância dos erros é uniforme, pois o teste não deu significância, confirmando a hipótese.

Após a análise do atendimento aos pressupostos mencionados, realizou-se a análise da regressão linear múltipla. A partir desta regressão buscou-se verificar se as variáveis propostas explicam o PIB dos municípios do Estado de Santa Catarina. A Tabela 2 apresenta o resumo do modelo da regressão linear múltipla.

Observa-se por meio da Tabela 3 que esta correlação é justificada pelo coeficiente de correlação (R) de 0,998, o que indica uma associação de 99,8\% entre as variáveis utilizadas no modelo. Já no que se refere ao coeficiente de determinação $\left(\mathrm{R}^{2}\right)$, serve como uma medida de quanto à variabilidade da saída pode ser atribuída aos previsores e apresenta um valor de 0,996, ou seja, as variáveis independentes explicam o 99,6\% do Produto Interno Bruto (PIB).

Tabela 3 - Resumo do modelo

\begin{tabular}{c|c|c|c|c}
\hline Modelo & $\mathbf{R}$ & $\mathbf{R}^{\mathbf{2}}$ & $\mathbf{R}^{\mathbf{2}}$ ajustado & Erro padrão da estimativa \\
\hline 1 & 0,998 & 0,996 & 0,995 & 114173,74 \\
\hline
\end{tabular}

Fonte: Dados da pesquisa.

O R ${ }^{2}$ ajustado obtido por meio da regressão fornece uma noção de quão bem o modelo generaliza os resultados e, quanto mais próximo for do valor do $\mathrm{R}^{2}$ melhor é o poder explicativo do modelo. Para tanto, obteve-se uma diferença de 0,001 entre o $\mathrm{R}^{2}(0,996)$ e o $\mathrm{R}^{2}$ ajustado $(0,995)$. Na sequência, por meio da Tabela 4 apresenta-se a análise da variância ANOVA, a qual objetiva testar se o modelo apresenta um poder explicativo melhor do que a média utilizada para explicar as variáveis independentes.

De acordo com os resultados obtidos por meio da Tabela 4, percebe-se que todas as variáveis apresentaram variâncias parecidas, ou seja, os dados são considerados homocedásticos. Para tanto, verifica-se que o modelo apresentou-se significante ao nível de $1 \%$, ou seja, as variáveis independentes utilizadas no estudo explicam o PIB e, nota-se que a 
relação entre as variáveis analisadas apresenta um alto poder explicativo para as variáveis selecionadas no modelo.

Tabela 4 - ANOVA

\begin{tabular}{c|l|c|c|c|c|c}
\hline \multicolumn{2}{c|}{ Modelo } & $\begin{array}{c}\text { Soma dos } \\
\text { Quadrados }\end{array}$ & gl & Quadrado Médio & F & Sig. \\
\hline \multirow{3}{*}{1} & Regressão & 83,45 & 4 & 20,86 & 16004,64 & 0,000 \\
\cline { 2 - 7 } & Erro & 37,41 & 287 & 13,03 & & \\
\cline { 2 - 7 } & Total & 83,82 & 291 & & & \\
\hline
\end{tabular}

Fonte: Dados da pesquisa.

Com o intuito de analisar a relação das variáveis independentes com a variável dependente, apresentam-se na Tabela 5, os coeficientes de regressão linear múltipla, para a amostra que compreende todos os municípios do Estado de Santa Catarina no ano de 2010.

Tabela 5 - Coeficientes do modelo de regressão

\begin{tabular}{l|c|c|c|c|c}
\hline \multirow{2}{*}{ Modelo } & \multicolumn{2}{|c|}{ Coeficientes não padronizados } & $\begin{array}{c}\text { Coeficientes } \\
\text { padronizados }\end{array}$ & \multirow{2}{*}{ T } & Sig. \\
\cline { 2 - 5 } & $\mathbf{B}$ & Modelo padrão & Beta & & 0,000 \\
(Constante) & 43114,548 & 10095,897 & & 4,271 & 0,000 \\
DVA AGRO & 1,470 & 0,238 & 0,025 & 6,168 & 0,000 \\
DVA IND & 1,066 & 0,022 & 0,356 & 48,380 & 0,000 \\
DVA SERV & 1,977 & 0,023 & 1,036 & 86,305 & 0,000 \\
DVA ADM & $-4,892$ & 0,189 & $-0,358$ & $-25,821$ & \\
\hline
\end{tabular}

Fonte: Dados da pesquisa.

Por meio da Tabela 5, é possível verificar quais as variáveis que influenciam no Produto Interno Bruto. Dessa forma, ao nível de significância de 1\% observa-se que todas as variáveis foram significantes, sendo estas a Demonstração do Valor Adicionado da Agropecuária, da Indústria, dos Serviços e ainda, a DVA da Administração. Todas as variáveis possuem influência positiva sobre o PIB, o que indica que quanto maior for o valor das DVAs, maiores serão os valores do PIB. Porém, nota-se que a DVA da Administração apresenta coeficiente negativo de 4,892 e, quando analisado o conjunto das DVAs, é a que menos está relacionada com o PIB, ou seja, quando os valores desta variável aumentam, consequentemente diminui o PIB, pois neste estudo apresentam-se inversamente proporcionais por ser um investimento que o município realiza. Na Tabela 6 apresenta-se as correlações do PIB com as quatro DVAs analisadas.

Tabela 6 - Correlação do PIB com as DVAs

\begin{tabular}{c|c|c|c|c}
\hline $\begin{array}{c}\text { Variável } \\
\text { dependente }\end{array}$ & DVA AGRO & DVA IND & DVA SERV & DVA ADM \\
\hline PIB & 0,0717 & 0,8692 & 0,9792 & 0,9223 \\
\hline
\end{tabular}

Fonte: Dados da pesquisa.

Por meio dos dados evidenciados na Tabela 6, nota-se que quando analisadas as variáveis independentes de forma individual com o PIB, a DVA da Agropecuária (DVA AGRO) foi a que menos teve relação, apresentando uma correlação de 0,0717 , o que representa $7,17 \%$ e a que mais demonstrou-se relacionada com o PIB foi a DVA dos Serviços (DVA SERV) com um valor de 0,9792, ou seja, 97,92\%. 
Diante disso, foi evidenciado a partir dos dados coletados, o percentual que cada DVA analisada corresponde ao valor total das DVAs que pode ser visualizada na Tabela 7.

Tabela 7 - Percentual de representatividade entre as DVAs

\begin{tabular}{l|c|c}
\hline \multicolumn{1}{c|}{ Variáveis } & TOTAL R\$ & TOTAL \% \\
\hline DVA AGRO & $\mathrm{R} \$ 8.753 .836,11$ & $6 \%$ \\
\hline DVA IND & $\mathrm{R} \$ 44.527 .902,03$ & $31 \%$ \\
\hline DVA SERV & $\mathrm{R} \$ 77.336 .159,69$ & $53 \%$ \\
\hline DVA ADM & $\mathrm{R} \$ 14.988 .166,60$ & $10 \%$ \\
\hline Total & $\mathbf{1 4 5 . 6 0 6 . 0 6 4 , 4 3}$ & $\mathbf{1 0 0 \%}$ \\
\hline
\end{tabular}

Fonte: Dados da pesquisa.

A partir dos dados da Tabela 7 verifica-se que a Demonstração de Valor Adicionado que apresentou um maior valor, considerando a soma de todos os municípios catarinenses é a DVA dos Serviços que apresenta um valor de 77.336.159,69, o que representa 53\% do total, valor bem acima da DVA de Agronegócios e da DVA da Administração, estas que na sua soma evidenciaram valores de 8.8753.836,11 e 14.988.166,60 respectivamente, o que representa $6 \%$ para a primeira e $10 \%$ para a segunda.

Diante disso, nota-se que devido ao fato de a DVA da Administração ter sido a única que no conjunto de variáveis apresentou-se significativa, porém com valor negativo e, àquela que faz com que o PIB diminua nos municípios analisados. Isso pode ocorrer em função da proporção de representatividade apresentada entre as DVAs analisadas. Ainda, a DVA do Agronegócio foi a variável que ao relacionar individualmente todas com o PIB, apresentou a menor relação. Já a DVA dos Serviços foi a variável que teve a maior relação com o PIB, o que corresponde ao mesmo resultado evidenciado na Tabela 7 , ou seja, conforme aumenta o valor da riqueza gerada pelos municípios catarinenses, qualquer que seja a DVA, aumenta o PIB.

\section{Considerações Finais}

O estudo teve por objetivo analisar a relação do Produto Interno Bruto dos municípios do Estado de Santa Catarina com as quatro Demonstrações do Valor Adicionado, sendo estas as DVAs de Agronegócios, Indústria, Serviços e Administração. Para tanto, realizou-se um estudo descritivo, de corte seccional com abordagem quantitativa dos 293 municípios catarinenses.

A partir dos resultados obtidos no estudo verificou-se que houve uma forte relação do Produto Interno Bruto (PIB) com todas as variáveis analisadas, sendo as Demonstrações do Valor Adicionado (DVA) do Agronegócio, da Indústria, dos Serviços e da Administração. Porém, verificou-se que a DVA da Administração, quando analisada no conjunto com as demais DVAs foi a que menos apresentou correlação com o PIB. Já quando analisadas individualmente, a DVA do Agronegócio foi o que menos se correlacionou. Isso se deve ao fato destas duas variáveis terem um menor valor quando somados todos os municípios em relação às demais DVAs analisadas.

Dessa forma, conclui-se que a Demonstração do Valor Adicionado é considerada uma importante aliada na formação do Produto Interno Bruto, pois conforme a literatura a DVA apresenta a parcela que os municípios contribuíram para formar o valor do PIB (CPC 09). É

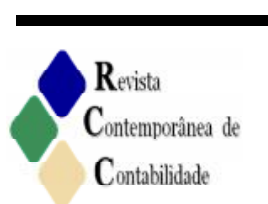

ISSN 2175-8069, UFSC, Florianópolis, v. 11, n. 24, p. 125-142, set./dez. 2014 
um instrumento capaz de evidenciar aspectos econômicos e sociais, constituindo uma das mais ricas demonstrações da contabilidade (CUNHA; RIBEIRO; SANTOS, 2005). Além disso, a DVA passa a ser um substituto para o lucro econômico (BAO; BAO, 1998). Têm sido apontada como um dos mais significativos instrumentos dentre as demonstrações contábeis obrigatórias (SIQUEIRA, 2007) para diagnosticar a situação da empresa (MORLEY, 1979).

Assim, percebe-se de acordo com os autores que, o PIB é considerado um índice macroeconômico para mensurar as atividades econômicas de determinadas regiões, municípios, Estados ou país durante certo período (BOULLOSA; TAVARES, 2009). Para tanto, de acordo Marion (1999) a DVA é uma demonstração útil, pois seu valor representa o $\mathrm{PIB}$, o que confirma o resultado encontrado neste estudo, pois todas as DVAs apresentaram relação significativa estatisticamente com o PIB dos municípios catarinenses analisados.

Este estudo contribui para confirmar o que a literatura aborda sobre a DVA e o PIB. Dessa forma, é importante a elaboração correta da Demonstração do Valor Adicionado (DVA), que conforme Cunha, Ribeiro e Santos (2005) é a mais rica demonstração contábil, pois apresenta a riqueza gerada pelos municípios, o que contribui para a formação do Produto Interno Bruto (PIB).

Como possibilidade para a realização de estudos futuros, recomenda-se efetuar uma análise longitudinal, ou seja, analisando mais anos. Além disso, a amostra do estudo poderá ser ampliada, visto que pode-se analisar uma região e, até mesmo todo país, o que possibilitará a utilização dos resultados encontrados nesta pesquisa, para fins de comparabilidade dos resultados.

\section{Referências}

Accounting Standards Steering Committee. The corporate report. London, 1975.

ALEXANDER, G. J.; BUCHHOLZ, R. A. Corporate social responsibility and stock market performance. Academy of Management Journal, v. 21, n. 3, p. 479-486, 1978.

ARAÚJO NETO, D. L. de; COSTA, E. de F. Dimensionamento do PIB do agronegócio em Pernambuco. Revista de Economia e Sociologia Rural, v. 43, n. 4, p. 725-757, 2005.

ARAÚJO, E.; FAJARDO, J.; TAVANI, L. C. di. CAPM usando uma carteira sintética do PIB Brasileiro. Estudos Econômicos - São Paulo, v. 36, n. 3, p. 465-505, 2006.

ASCAHUER, D. Is public expenditure productive? Journal of Monetary Economics, v. 23, p. 177-200, 1989.

ASWEGEN, N. V.; STEYN, B. W.; HAMMAN, W. D. Trends in the distribution of added value of listed industrial companies - 1990 to 2002. South African Journal of Business Management, v. 36, n. 2, p. 85-94, 2005.

BAO, B.-H.; BAO, D.-H. Usefulness of value added and abnormal economic earnings: an empirical examination. Journal of Business Finance \& Accounting, v. 25, n. 1-2, p. 251 264, 1998. 
BARRO, R. A. Government spending in a simple model of endogenous growth. Journal of Political Economy, v. 98, p. 1-57, 1990.

BENTLEY, T. Added value and contribution. Management Accounting, v. 59, n. 3, p. 7-21, 1981.

BOULLOSA, R.; TAVARES, E. Avaliação e monitoramento de projetos sociais. Curitiba: IESDE Brasil S.A., 2009.

BRASIL. Lei no 11.638, de 28 de dezembro de 2007. Dispõe sobre as Sociedades por Ações. Diário Oficial, República Federativa do Brasil, Brasília - DF, 2007. Disponível em: $<$ http://www.planalto.gov.br/ccivil_03/_ato2007-2010/2007/lei/111638.htm>. Acesso em: 08 Jun. 2014.

BURCHELL, S.; CLUBB; C.; HOPWOOD, A. G. Accounting and its Social Context: Towards a History of Value Added in the United Kingdom. Accounting, Organizations and Society, v.10, n. 4, p.381-413, 1985.

CASHIN, P. Government spending taxes and economic growth. IMF Staff Papers, v. 42, n. 2, p. 237-269, 1995.

CHUA, K. C. The Use of Value Added in Productivity Measurements. In Productivity Measurement and Achievement: Proceedings of Accountancy. Victoria: University of Wellington, 1977.

Comitê de Pronunciamentos Contábeis. Pronunciamento técnico CPC 09: Demonstração do Valor Adicionado, 2008. Disponível em: <http://www.cpc.org.br/CPC/DocumentosEmitidos/Pronunciamentos/Pronunciamento?Id=40> Acesso em: 10 Jun. 2014.

COSENZA, J. P. A eficácia informativa da demonstração do valor adicionado. Revista Contabilidade \& Finanças, v. 14, p. 7-29, 2003.

CUNHA, J. V. A. da; RIBEIRO, M. de S.; SANTOS, A. dos. A demonstração do valor adicionado como instrumento de mensuração da distribuição da riqueza. Revista Contabilidade \& Finanças, v. 16, n. 37, p. 7-23, 2005.

EVRAERT, S.; RIAHI-BELKAOUI, A. Usefulness of Value Added Reporting: a review and synthesis of the literature. Managerial Finance, v. 24, n. 11, p. 1-15, 1998.

FÁVERO, L. P.; BELFIORE, P.; SILVA, F. L. da; CHAN, B. L.. Análise de Dados: modelagem multivariada para a tomada de decisões. Rio de Janeiro: Elsevier, 2009.

FURTUOSO, M. C. O.; GUILHOTO, J. M. Estimativa e mensuração do Produto Interno Bruto do Agronegócio da Economia Brasileira, 1994 a 2000. Revista de Economia e Sociologia Rural, v. 41, n. 4, p. 803-827, 2003. 
GASTALDI, J. P. Elementos de economia política. 19. ed. São Paulo: Saraiva, 2005.

HAIR, Jr., J. F.; ANDERSON, R. E.; TATHAM, R. L.; BLACK, W. C.. Análise multivariada de dados. 5. ed. Porto Alegre: Bookman, 2005.

HAIR, Jr., J. F.; BLACK, W. C.; BABIN, B. J.; ANDERSON, R. E.; TATHAM, R. L.; Análise multivariada de dados. 6. ed. Porto Alegre: Bookman, 2009.

HALLER, A.; STOLOWY, H. Valued added in financial accounting, a comparative study of Germany and France. Advances in international accounting, v. 11, p. 23-51, 1998.

HEILBRONER, R. L.; THUROW, L. Entenda a economia: tudo que você precisa saber sobre como funciona e para onde vai a economia. Rio de Janeiro: Campus, 2001.

Instituto Brasileiro de Geografia e Estatística (IBGE). Estatísticas - PIB municípios, 2010. Disponível em: $<$ http://downloads.ibge.gov.br/downloads_estatisticas.htm $>$. Acesso em: 12 Abr. 2014.

IOAN, Done. Employment-Cause and Effect of the Economic Growth. Procedia Economics and Finance, v. 8, p. 268-274, 2014.

KENNEDY, Peter. A guide to econometrics. Cambridge: The MIT Press, 1998.

KNELL, A. Added value for added profits. London: The Institute of Chartered Accountants in England and Wales, 1986.

KROETZ, C. E. S. Balanço social, teoria e prática. São Paulo: Atlas, 2000.

LUCA, M. M. M. de. Demonstração do Valor Adicionado: do cálculo da riqueza criada pela empresa. São Paulo: Atlas, 1998.

MARION, J. C. Demonstrações financeiras/contábeis: propostas para alteração da Lei das Sociedades Anônimas. Contabilidade Vista \& Revista, v. 10, n. 3, p. 23-38, 1999.

. Análise das Demonstrações Contábeis - Contabilidade Empresarial. São Paulo: Atlas, 2001.

MAROCO, J. Análise Estatística com o SPSS statistics. $5^{\text {a }}$ ed. Pero Pinheiro: Report Number, 2011.

MARTINS, E.; GELBCKE, E. R.; SANTOS, A. dos; IUDÍCIBUS, S. de. Manual de Contabilidade Societária: Aplicável a todas as Sociedades de acordo com as Normas Internacionais de Contabilidade e do CPC. FIPECAFI. 2. ed. São Paulo: Atlas, 2013. 
MARTINS, G. de A.; THEÓPHILO, C. R. Metodologia da investigação científica para ciências sociais aplicadas. 2. ed. São Paulo: Atlas, 2009.

MEEK, G. K.; GRAY, S. J. The value added statement: an innovation for U.S. companies? Accounting Horizons, p.73-81, 1988.

MORLEY, M. F. The Value Added Statement in Britain. The accounting review, n. 3, p. 618-629, 1979.

PENDRILL, D. Introducing a New comer: The Value Added Statement. Accountancy, v. 88, n. 1012, p. 92-94, 1977.

PICUR, R. D. The effects of accounting knowledge on the omission of value added information in wealth measurement and distribution decisions. Review of Accounting and Finance, v. 6, n. 1, p. 15-23, 2007.

PINTO, A. L.; RIBEIRO, M. de S. Balanço social: avaliação de informações fornecidas por empresas industriais situadas no estado de Santa Catarina. Revista Contabilidade \& Finanças, v. 15, n. 36, p. 21-34, 2004.

PONG, C.; MITCHELL, F. Accounting for a disappearance: a contribution to the history of the value added statement in the UK. Accounting Historians Journal, v. 32, n. 2, p. 173199, 2005.

RAM, R. Government size and economic growth: a new framework and some evidence from cross-section and time series data. American Economic Review, v. 76, n. 1, p.281-284, 1989.

RIAHI-BELKAOUI, A. The New Environment in Internacional Accounting. Quorum Books, 1998.

ROSSETTI, J. P. Introdução à economia. 20. ed. São Paulo: Atlas, 2003.

SACHS, J. D.; LARRAIN B. F. Conceitos Básicos de Macroeconomia. São Paulo: Pearson Makron Books, 2000.

SAMPIERI, R. H.; COLLADO, C. F.; LUCIO, P. B.; PÉREZ, M. D. L. L. C. Metodologia de la investigación. México: Mcgraw-Hill, 1991.

SANTOS, A.; HASHIMOTO, H. Demonstração do valor adicionado: algumas considerações sobre carga tributária. Revista de Administração, v. 38, n. 2, p. 153-164, 2003.

SIMONSEN, Mário Henrique. Macroeconomia. 7. ed. Rio de Janeiro: Apec, 1979.

SIQUEIRA, J. R. M. DVA: vantagens e limitações de seu uso como instrumento de avaliação da estratégia social corporativa. Revista Del Instituto Internacional de Costos, v. 1, n. 1, p. 116-140, 2007. 
STAINBANK, L. J. The value added statement: does it add any value? Meditari Accountancy Research, v. 17, n. 2, p. 137-149, 2009.

SUOJANEN, W. W. Accounting theory and the large corporation. The Accounting Review, p. 391-398, 1954.

TINOCO, J. E. P. Balanço social: Uma abordagem socioeconômica da contabilidade. Dissertação (Mestrado em Economia e Administração). Universidade de São Paulo Faculdade de Economia e Administração, São Paulo: FEA/USP, 1984.

ULLMANN, A. A. Data in search of a theory: a critical examination of the relationships among social performance, social disclosure, and economic performance of U.S. firms. Academy of management review, v. 10, n. 3, p. 540-557, 1985.

VAN STADEN, C. J. The value added statement: bastion of social reporting or dinosaur of financial reporting? Palmerston North, NZ: School of Accountancy, Massey University, 2000.

VERGARA, S. C. Projetos e relatórios de pesquisa em administração. 2. ed. São Paulo: Atlas, 1998. 
